\title{
RENORMALIZATION GROUP EVOLUTION OF MULTI-GLUON CORRELATORS IN HIGH ENERGY QCD
}

\author{
JAMAL JALILIAN-MARIAN \\ Natural Sciences Department, Baruch College, 17 Lexington Ave., \\ New York, NY 10010, USA \\ jamal.jalilian-marian@baruch.cuny.edu
}

\begin{abstract}
Forward rapidity di-hadron azimuthal angular correlations in high energy proton-nucleus and proton-proton collisions are sensitive to quadrupoles; traceless correlator of 4 Wilson lines whereas single inclusive particle production iNVOLVES only dipoles, traceless correlator of 2 Wilson lines. We discuss the progress made in understanding the energy (rapidity) evolution of the quadrupole as well as its various limits.

Keywords: High energy QCD; Color Glass Condensate; Quadrupoles; di-hadron angular correlations.
\end{abstract}

\section{Introduction}

The past decade has seen much progress made in understanding particle production in high energy collisions using the Color Glass Condensate (CGC) formalism. Among the phenomena investigated are hadron multiplicities as a function of rapidity in heavy ion collisions, single inclusive particle production and two-particle correlations (azimuthal angular or rapidity) in proton-proton and proton-nucleus collisions. $^{1}$

The Color Glass Condensate formalism ${ }^{2}$ is an effective action approach to the high gluon density environment, expected to occur in the wave function of a hadron or nucleus at high energy. Whereas the hadron (nucleus) wave function compromises of the constituent quarks and a few sea quarks and gluons at large $x$, it is expected to contain a large number of sea quarks and gluons at small $x$ where $x \equiv \frac{Q^{2}}{S}$ is the fraction of the hadron energy carried by a parton (quark or gluon). The standard perturbative QCD (leading twist) techniques fail in this case since, roughly speaking, all twist contributions are of the same order so that a twist expansion can not be valid. Therefore, a new formalism which can take high gluon density effects is needed. This high gluon density environment can be reached by boosting the hadron/nucleus to higher energies which increases the longitudinal phase space available (at fixed transverse momenta) for parton radiation. Since QCD splitting functions (gluon channel) are singular as $x \rightarrow 0$, radiation of soft (longitudinal momenta) are favored which lead to a sharp increase of the number of gluons in the hadron/nucleus wave function. Thus any formalism attempting to address the 
dynamics of this high gluon density environment must include a re-summation of the large energy logs that appear. This re-summation typically goes under the name of $\mathrm{BFKL}^{3}$ re-summation. In addition to re-summing large logs of energy, one must also include "multiple scattering" effects since they may be important due to large gluon densities.

In the CGC approach, one separates large and small $x$ degrees of freedom. This is due to the vastly different intrinsic time scales involved. The natural time scale for large $x$ degrees of freedom is $\tau_{0}$ which is much larger than the time scale of the large $x$ degrees of freedom which is $x \tau_{0}$. Therefore, the small $x$ degrees of freedom see the the color charges $\rho$ (representing the large $x$ degrees of freedom) as frozen in time, i.e., static. One therefore starts with an effective action which treats the large $x$ degrees of freedom as static (Light Cone time $x^{+}$independent) color charges $\rho\left(x_{t}\right) \equiv \rho^{2}\left(x_{t}\right) T^{a}$. On the other hand, the small $x$ degrees of freedom are described by the standard gluon field $A^{\mu}(x)$ which are generated by the color charges $\rho$ and satisfy the classical equations of motion

$$
D_{\mu} F_{a}^{\mu \nu}(x)=J_{a}^{\nu}(x)
$$

where $J_{a}^{\nu}(x)=g \delta^{\nu+} \rho_{a}\left(x_{t}, x^{-}\right)$is the color current of large $x$ degrees of freedom. As a first step, one solves the equation of motion for the classical gluon field $A_{\mu}^{a}$ which, in the covariant gauge, gives $A_{a}^{\mu}=\delta^{\mu+} \alpha_{a}\left(x_{t}, x^{-}\right)$with $\alpha_{a}\left(x_{t}, x^{-}\right)=-\frac{1}{\partial_{t}^{2}} \rho_{a}\left(x_{t}, x^{-}\right)$. With these approximations in mind, the effective action can be written as

$$
\begin{aligned}
S \equiv & -\frac{1}{4} \int d^{4} x F_{\mu \nu}^{a 2}(x)+\frac{i}{N_{c}} \int d^{2} x d x^{-} \operatorname{Tr} \rho\left(x^{-}, x_{t}\right) W_{-\infty, \infty}\left[A^{-}\right]\left(x^{-}, x_{t}\right) \\
& +i \int d^{2} x d x^{-} F\left[\rho\left(x_{t}, x^{-}\right)\right],
\end{aligned}
$$

where

$$
W_{-\infty, \infty}\left[A^{-}\right]\left(x^{-}, x_{t}\right) \equiv P \exp \left[-i g \int_{-\infty}^{\infty} d x^{+} A_{a}^{-}\left(x^{+}, x^{-}, x_{t}\right) T_{a}\right]
$$

and $\exp \left[-\int d^{2} x d x^{-} F\left[\rho\left(x_{t}, x^{-}\right)\right]\right]$can be thought of as the statistical weight of a given color charge configuration. Since the color charges $\rho$ are effective degrees of freedom introduced to represent the large $x$ degrees of freedom, one needs to average over them with this statistical weight when computing physical observables. The function $F$ was taken to be of a Gaussian form in the McLerran-Venugopalan model such that

$$
\int d x^{-} F\left[\rho\left(x_{t}, x^{-}\right)\right]=\frac{1}{2 \mu^{2}} \rho^{2}\left(x_{t}\right) .
$$

This Gaussian form follows from intuitive reasoning and can be derived from group theory. It is however not exact and receives corrections, in the form of $\rho^{3}, \rho^{4}$ which can be important for certain observables. ${ }^{4}$ Lastly, one should keep in mind that 
the longitudinal structure of the color charge $\rho$ is important when one wants to compute averages of the color charge correlators at the same rapidity $(x)$. On the other hand, if one is interested in the energy dependence (evolution) of correlators, one can treat this longitudinal structure as being a delta function of $x^{-}$.

\section{Particle Production in CGC Formalism}

Color Glass Condensate formalism has been applied to particle production in high energy proton-proton, proton-nucleus and nucleus-nucleus collisions and well as in DIS. Here we briefly review some of the most common assumptions and approximations involved.

\subsection{Multiplicities}

To calculate hadron multiplicities (mid-rapidity) in heavy ion (symmetric) collisions, one needs to find the color field produced after the nuclei collide (in the forward Light Cone). To do this, one uses the known one-nucleus solution (given above) as the initial condition for the collision of two nuclei. One then needs to solve the equation of motion subject to this initial condition and find the color field produced in the mid-rapidity region after the collision. Unfortunately, the equations involved are too complicated and an analytic solution has not been found. Alternatively one can solve the equations of motion numerically (on a lattice) and compute particle number and energy densities produced in the early times after the collision. A complication, that arises when trying to compute the observed hadron multiplicities in a nucleus-nucleus collision, is the fact that a Quark-Gluon Plasma is produced which can in principle affect the particle number and energy distributions. However, due to entropy conservation and that the produced plasma seems to be interacting, it seems to be a good approximation to simply relate the number of produced partons at the early stage of a high energy heavy ion collision with that of observed hadrons. Therefore, for practical applications, one uses the following " $k_{t}$ " factorized form to calculate hadron multiplicities, ${ }^{5}$

$$
\frac{d N}{d y}=\int d^{2} p_{t} d^{2} k_{t} \Phi\left(y, p_{t}\right) \Phi\left(y,\left(p_{t}-k_{t}\right)\right)
$$

where $\Phi\left(x, p_{t}\right)$ is the unintegrated gluon distribution function which becomes the standard gluon distribution function upon integrating over the intrinsic momentum $p_{t}$. The function $\Phi$ satisfies the non-linear $\mathrm{BK}^{6}$ (or JIMWLK) equation which includes high gluon density effects. Lastly, it should be mentioned that the use of Eq. (4) for parton production in symmetric (nucleus-nucleus or proton-proton) collisions may be justified only at high transverse momenta whereas multiplicities are dominated by low $p_{t}$. The same equation is used to compute multiplicities in proton-proton and proton-nucleus collisions. 


\subsection{Single inclusive spectra}

As mentioned above, transverse momentum and rapidity spectra of partons produced in the earlies moments after a high energy heavy ion collision can be modified due to the presence of a QGP. However, one does not expect to produce a QGP in a proton-nucleus or proton-proton collision where final state effects may be negligible. Therefore, we focus on proton-proton and proton-nucleus collisions in the rest of this article. In the mid-rapidity region and at high energy where $x_{1,2} \sim \frac{p_{t}}{\sqrt{s}} e^{ \pm y} \ll 1$ one can use Eq. (4) to compute transverse momentum spectra, $d N y d^{2} p_{t}$, of hadrons produced in single inclusive processes. On the other hand, as one goes to more forward rapidities, the $k_{t}$ factorized form breaks down since the projectile $x$ is not small anymore. One therefore needs to use a "hybrid" formalism ${ }^{7}$ where one treats the projectile (proton) as a collection of partons, as in pQCD, while the target (proton or nucleus) is probed at small $x$ and can therefore be treated by CGC techniques. The single inclusive production cross section is now given by

$$
\begin{aligned}
\frac{d \sigma^{p A \rightarrow h X}}{d y d^{2} p_{t} d^{2} b}= & \frac{1}{(2 \pi)^{2}} \int_{x_{F}}^{1} d x \frac{x}{x_{F}}\left\{f_{q / p}\left(x, Q^{2}\right) N_{F}\left[\frac{x}{x_{F}} p_{t}, b\right] D_{h / q}\left(\frac{x_{F}}{x}, Q^{2}\right)\right. \\
& \left.+f_{g / p}\left(x, Q^{2}\right) N_{A}\left[\frac{x}{x_{F}} p_{t}, b\right] D_{g / h}\left(\frac{x_{F}}{x}, Q^{2}\right)\right\},
\end{aligned}
$$

where $y$ and $p_{t}$ are the rapidity and transverse momentum of the produced hadron and $x_{F}$ is Feynman- $x$. The factorization/renormalization scale $Q^{2}$ is typically taken to be the transverse momentum of the produced hadron. The underlying picture leading to this "hybrid" equation is simple; at the Leading Order, a quark or gluon from the projectile proton scatters on the classical color field of the nucleus and then hadronizes. Including loop corrections, with leading log accuracy, leads to the scale dependence of the quark and gluon distribution of the projectile proton. The (fundamental or adjoint) dipole cross section is denoted by $N_{F}, N_{A}$ and can be understood at arise as following; the amplitude for (eikonal) scattering of a quark or gluon on the background color field involves a Wilson line $V(U)$ which upon squaring gives the dipole cross section $N_{F}\left(N_{A}\right)$ which satisfies the BK/JIMWLK evolution equation.

There are two recent developments which are important and should be mentioned; first higher order corrections to Eq. (5) have been computed, ${ }^{8}$ second, full NLO corrections to the dipole evolution equation now exist. It is now standard to use the solution to the BK equation with running coupling corrections ${ }^{9}$ included which results in excellent agreement of the predictions of this approach with the experimental data.

\subsection{Di-hadron angular correlations in proton (deuteron)-nucleus collisions}

To further probe saturation physics and to map out its region of applicability, twoparticle angular correlations have been proposed ${ }^{10,11}$ and measured recently. ${ }^{12}$ The 
observed disappearance of the away side peak in forward rapidity di-hadron angular correlation with decreasing transverse momenta has lead to much excitement in the field and generated renewed interest in the predictions of CGC formalism for this observable. To investigate this observable in this kinematics, we remember that, similar to single inclusive production, one is probing the large $x$ region of the projectile wave function but the small $x$ region of the target wave function. One therefore starts with a quark from the projectile scattering on the classical color field of the nucleus. This quark can radiate a gluon either before or after the scattering from the target color field. Both quark and gluon are then produced, i.e. hadronize and are measured. For completeness one needs to include gluons from the projectile wave function also but this does not change the essential underlying physics. The disappearance of the away side has a simple interpretation, it is a combination of two effects, first multiple scattering of either quark, gluon or both will change the angle between the two away from back to back configuration, and second, the target wave function will be depleted of gluons (as compared to number of gluons in $A$. Nucleons, referred to as nuclear shadowing ${ }^{13}$ ) due to small $x$ evolution of the target wave function. The width and height of the away side peak will then depend on the strength of the multiple scattering and $x$ dependence of the scattering probability.

The cross section for two hadron production will then involve products of four Wilson lines (called a quadrupole). Therefore, we define the quadrupole operator as

$$
Q(r, \bar{r}, \bar{s}, s) \equiv \frac{1}{N_{c}} \operatorname{tr} V_{r} V_{\bar{r}}^{\dagger} V_{\bar{s}} V_{s}^{\dagger},
$$

where $V_{r} \equiv V\left(r_{t}\right)$ is a Wilson line in the fundamental representation in the covariant gauge

$$
V\left(r_{t}\right) \equiv \hat{P} e^{-i g \int d x^{-} A^{+}}
$$

Appearance of four fundamental Wilson lines may seem a little strange since gluons are described by a Wilson line in the adjoint representation, however, this follows after some trivial color algebra and using the following relation between fundamental and adjoint matrices $[U(r)]^{a b} t^{b}=V^{\dagger}(r) t^{a} V(r)$.

It is important to notice that unlike single inclusive particle production which involved dipoles only, traceless product of two Wilson lines, di-hadron production cross section contains higher number of Wilson lines. This is the first process where higher $n$-point functions of CGC appear. Whereas properties of the dipole are well known in various limits, not much is known about quadrupoles. An evolution equation for the quadrupole has been derived and its various limits are being investigated. ${ }^{10,14,15}$ The quadrupole evolution equation is

$$
\begin{aligned}
& \frac{d}{d y}\langle Q(r, \bar{r}, \bar{s}, s)\rangle=\frac{N_{c} \alpha_{s}}{(2 \pi)^{2}} \int d^{2} z \\
& \quad \times\left\{\left\langle\left[\frac{(r-\bar{r})^{2}}{(r-z)^{2}(\bar{r}-z)^{2}}+\frac{(r-s)^{2}}{(r-z)^{2}(s-z)^{2}}-\frac{(\bar{r}-s)^{2}}{(\bar{r}-z)^{2}(s-z)^{2}}\right] Q(z, \bar{r}, \bar{s}, s) S(r, z)\right.\right.
\end{aligned}
$$




$$
\begin{aligned}
& +\left[\frac{(r-\bar{r})^{2}}{(r-z)^{2}(\bar{r}-z)^{2}}+\frac{(\bar{r}-\bar{s})^{2}}{(\bar{r}-z)^{2}(\bar{s}-z)^{2}}-\frac{(r-\bar{s})^{2}}{(r-z)^{2}(\bar{s}-z)^{2}}\right] Q(r, z, \bar{s}, s) S(z, \bar{r}) \\
& +\left[\frac{(\bar{r}-\bar{s})^{2}}{(\bar{r}-z)^{2}(\bar{s}-z)^{2}}+\frac{(s-\bar{s})^{2}}{(s-z)^{2}(\bar{s}-z)^{2}}-\frac{(\bar{r}-s)^{2}}{(s-z)^{2}(\bar{r}-z)^{2}}\right] Q(r, \bar{r}, z, s) S(\bar{s}, z) \\
& +\left[\frac{(r-s)^{2}}{(r-z)^{2}(s-z)^{2}}+\frac{(s-\bar{s})^{2}}{(s-z)^{2}(\bar{s}-z)^{2}}-\frac{(r-\bar{s})^{2}}{(r-z)^{2}(\bar{s}-z)^{2}}\right] Q(r, \bar{r}, \bar{s}, z) S(z, s) \\
& -\left[\frac{(r-\bar{r})^{2}}{(r-z)^{2}(\bar{r}-z)^{2}}+\frac{(s-\bar{s})^{2}}{(s-z)^{2}(\bar{s}-z)^{2}}+\frac{(r-s)^{2}}{(r-z)^{2}(s-z)^{2}}\right. \\
& -\left[\frac{(\bar{r}-\bar{s})^{2}}{(r-s)^{2}}\right] Q(r, \bar{r}, \bar{s}, s) \\
& -\left[\frac{\left.(\bar{r}-\bar{s})^{2}-z\right)^{2}}{(r-z)^{2}(s-z)^{2}}+\frac{(\bar{r}-s)^{2}}{(\bar{r}-z)^{2}(\bar{s}-z)^{2}}-\frac{(r-\bar{s})^{2}}{(\bar{r}-z)^{2}(s-z)^{2}}\right. \\
& \\
& -\overline{(r-z)^{2}(\bar{r}-z)^{2}}+\frac{(s-\bar{s})^{2}(\bar{s}-z)^{2}}{(s-z)^{2}(\bar{s}-z)^{2}}-\frac{(r-\bar{s})^{2}}{(r-z)^{2}(\bar{s}-z)^{2}}
\end{aligned}
$$

and the $S$ matrix is defined as

$$
S(r, \bar{r}) \equiv \frac{1}{N_{c}} \operatorname{tr} V_{r} V_{\bar{r}}^{\dagger}
$$

Despite its complicated looking form, Eq. (8) has a simple meaning; a quadrupole can evolve into a quadrupole and a dipole, remain a quadrupole or break into two dipoles. The emission kernel is the usual dipole kernel for a given configuration but unlike the dipole evolution, the coefficients are not positive definite. Approximate solutions in the Guassian approximation have been proposed. ${ }^{10,16}$ Recently, this equation has been solved numerically and a comparison is made with various approximate results. ${ }^{17}$

It has been shown that the quadrupole evolution equation reduces to a collection of BFKL equations for the evolution of a dipole when one expands the Wilson lines in powers of the gauge field (or color charge $\rho$ ) and keeps only the first non-trivial terms in the expansion in two of the Wilson lines, i.e. terms of order $\rho^{2}$. This expansion is valid when the external momenta of the produced hadrons are much larger than the saturation scale involved. This is the kinematics where single scattering dominates which leaves us to conclude that the produced hadrons are back to back, i.e. the away side peak is present at high transverse momenta.

To investigate the disappearance of the away side peak, we note that it happens when one lowers the transverse momenta of the hadrons. This corresponds to 
going beyond single scattering limit, the first correction to which is double scattering. We note that since we are interested in the first correction to single scattering at high transverse momenta, one can still neglect the non-linear terms in the quadrupole evolution equation. However, we now need to keep terms of order $\rho^{4}$. This involves straightforward but long algebra. The resulting equation for the quadrupole is shown ${ }^{15}$ to be identical to the BJKP evolution equation ${ }^{18}$ for the singlet state of 4-Reggeized gluons. Thus at high transverse momenta, exchange of 4-Reggeized gluons is responsible for disappearance of the away side peak in dihadron angular correlations in the forward rapidity region in deuteron-gold collisions at RHIC.

As one lowers the transverse momenta further, one expects that higher order terms in $\rho$ make bigger and bigger contributions. This corresponds to allowing higher number of Reggeized gluon exchanges which are also subject to the BJKP equation. One would also need to include, at some point, the non-linear terms in the evolution equation for the quadrupole make a significant contribution. In that case one would need to keep terms of order $\rho^{4}$ which are coming from the nonlinear terms. It is expected that this would correspond to the triple pomeron vertex contribution. ${ }^{19}$ Again, as the di-hadron transverse momenta are lowered, one would need to keep all pomeron vertices. The formal correspondence between the CGC formalism and multi-Reggeized gluon exchanges supplemented by triple (or more) pomeron vertices seems reasonable and will be reported on elsewhere.

It is worth mentioning that the complications arising in di-hadron angular correlations due to the higher point functions of Wilson lines can be avoided by considering photon-hadron angular correlations. ${ }^{20}$ This process involves only dipoles as the radiated photon does not strongly interact with the target nucleus. It is shown that all qualitative features of di-hadron angular correlations are also present in photon-hadron correlations.

\section{Acknowledgments}

This work is supported by the DOE Office of Nuclear Physics through Grant No. DE-FG02-09ER41620 and by The City University of New York through the PSCCUNY Research Program, grant 62625-42.

\section{References}

1. F. Gelis, E. Iancu, J. Jalilian-Marian, R. Venugopalan, Ann. Rev. Nucl. Part. Sci. 60, 463-489 (2010); J. Jalilian-Marian, Y. V. Kovchegov, Prog. Part. Nucl. Phys. 56, 104-231 (2006).

2. J. Jalilian-Marian, A. Kovner, L. D. McLerran and H. Weigert, Phys. Rev. D 55, 5414 (1997); J. Jalilian-Marian, A. Kovner, A. Leonidov and H. Weigert, Nucl. Phys. B 504, 415 (1997), Phys. Rev. D 59, 014014 (1999), Phys. Rev. D 59, 014015 (1999), Phys. Rev. D 59, 034007 (1999), A. Kovner, J. G. Milhano and H. Weigert, Phys. Rev. D 62, 114005 (2000); A. Kovner and J. G. Milhano, Phys. Rev. D 61, 014012 (2000); E. Iancu, A. Leonidov and L. D. McLerran, Nucl. Phys. A 692, 583 (2001), Phys. 
Lett. B 510, 133 (2001); E. Ferreiro, E. Iancu, A. Leonidov and L. McLerran, Nucl. Phys. A 703, 489 (2002); H. Weigert, Nucl. Phys. A703, 823-860 (2002). J.-P. Blaizot, E. Iancu, H. Weigert, Nucl. Phys. A713, 441-469 (2003).

3. L. N. Lipatov, Sov. J. Nucl. Phys. 23, 338 (1976) [Yad. Fiz. 23, 642 (1976)]; E. A. Kuraev, L. N. Lipatov and V. S. Fadin, Sov. Phys. JETP 45, 199 (1977) [Zh. Eksp. Teor. Fiz. 72, 377 (1977)]; I. I. Balitsky and L. N. Lipatov, Sov. J. Nucl. Phys. 28, 822 (1978) [Yad. Fiz. 28, 1597 (1978)].

4. A. Dumitru, J. Jalilian-Marian, E. Petreska, Phys. Rev. D84, 014018 (2011).

5. D. Kharzeev, E. Levin and M. Nardi, Phys. Rev. C 71, 054903 (2005).

6. I. Balitsky, Nucl. Phys. B 463, 99 (1996); Y. V. Kovchegov, Phys. Rev. D 60, 034008 (1999); Phys. Rev. D 61, 074018 (2000).

7. A. Dumitru, A. Hayashigaki and J. Jalilian-Marian, Nucl. Phys. A 765, 464 (2006). Nucl. Phys. A 770, 57 (2006).

8. T. Altinoluk and A. Kovner, Phys. Rev. D 83, 105004 (2011); G. A. Chirilli, B.W. Xiao and F. Yuan, Phys. Rev. D 86, 054005 (2012).

9. J. L. Albacete and Y. V. Kovchegov, Phys. Rev. D 75, 125021 (2007); J. JalilianMarian and A. H. Rezaeian, Phys. Rev. D 85, 014017 (2012). J. L. Albacete, A. Dumitru, H. Fujii and Y. Nara, arXiv:1209.2001 [hep-ph].

10. J. Jalilian-Marian and Y. V. Kovchegov, Phys. Rev. D 70, 114017 (2004) [Erratumibid. D 71, 079901 (2005)].

11. C. Marquet, Nucl. Phys. A 796, 41 (2007); K. Tuchin, arXiv:0912.5479 [hep-ph]; J. L. Albacete and C. Marquet, arXiv:1005.4065 [hep-ph]; D. Kharzeev, E. Levin and L. McLerran, Nucl. Phys. A 748, 627 (2005); A. Stasto, B.-W. Xiao and F. Yuan, arXiv:1109.1817 [hep-ph]; A. Kovner, M. Lublinsky, Phys. Rev. D83, 034017 (2011), [arXiv:1012.3398 [hep-ph]]; [arXiv:1109.0347 [hep-ph]]; R. Baier, A. Kovner, M. Nardi and U. A. Wiedemann, Phys. Rev. D 72, 094013 (2005).

12. E. Braidot [ STAR Collaboration ], Nucl. Phys. A854, 168-174 (2011); A. Adare et al. [PHENIX Collaboration], Phys. Rev. Lett. 107, 172301 (2011).

13. J. Jalilian-Marian and X.-N. Wang, Phys. Rev. D 63, 096001 (2001).

14. F. Dominguez, A. H. Mueller, S. Munier, B.-W. Xiao, Phys. Lett. B705, 106111 (2011); A. Dumitru and J. Jalilian-Marian, Phys. Rev. D 81, 094015 (2010), A. Dumitru, J. Jalilian-Marian, Phys. Rev. D82, 074023 (2010).

15. J. Jalilian-Marian, Phys. Rev. D 85, 014037 (2012).

16. F. Dominguez, C. Marquet, B.-W. Xiao, F. Yuan, Phys. Rev. D83, 105005 (2011); E. Iancu, D. N. Triantafyllopoulos, [arXiv:1109.0302 [hep-ph]].

17. A. Dumitru, J. Jalilian-Marian, T. Lappi, B. Schenke, R. Venugopalan, [arXiv:1108.4764 [hep-ph]].

18. J. Bartels, Nucl. Phys. B 175, 365 (1980); T. Jaroszewicz, Acta Phys. Polon. B 11, 965 (1980); J. Kwiecinski and M. Praszalowicz, Phys. Lett. B 94, 413 (1980).

19. G. A. Chirilli, L. Szymanowski and S. Wallon, Phys. Rev. D 83, 014020 (2011).

20. J. Jalilian-Marian and A. H. Rezaeian, Phys. Rev. D 86, 034016 (2012); J. JalilianMarian, Eur. Phys. J. C 61, 789 (2009); Nucl. Phys. A 770, 210 (2006). 